\title{
Incidence of deep vein thrombosis after major spine surgeries with no mechanical or chemical prophylaxis
}

Author Sreedharan Namboothiri

Institution Kovai Medical Center and Hospitals, Coimbatore, India

\begin{tabular}{l|l|}
\hline Final class of & \\
evidence-prognosis & \\
\hline Study design & \\
\hline Prospective cohort & \\
\hline Retrospective cohort & $\circ$ \\
\hline Case control & \\
\hline Case series & \\
\hline Methods & \\
\hline Patients at similar point in & \\
\hline course of treatment & \\
\hline F/U $\geq 85 \%$ & \\
\hline Similarity of treatment & \\
protocols for patient groups & \\
\hline Patients followed up long & $\circ$ \\
enough for outcomes to occur & \\
\hline Control for extraneous risk & \\
\hline factors & \\
\hline Overall class of evidence & III \\
\hline The definiton of the different \\
\hline classes of evidence is available \\
\hline on page 63. \\
\hline
\end{tabular}

\section{ABSTRACT}

Study design: Retrospective cohort study.

Clinical question: What is the incidence of deep vein thrombosis (DVT) and pulmonary embolism (PE) after major spine surgery when no prophylactic measures were used?

Methods: A prospective evaluation of 121 patients who underwent 128 major spine surgeries was conducted to determine the incidence of clinically identifiable DVT. As a matter of practice, no patient was given thromboprophylaxis, either mechanical or chemical.

Results: Only one patient developed the signs and symptoms of DVT, which was further confirmed by a Doppler study. The overall incidence of DVT was $0.78 \%$. There was no clinically evident case of PE.

Conclusions: Considering the low rate of incidence of DVT and PE, routine screening and prophylaxis for DVT appears unwarranted in major spine surgery.

The author has no conflict of interest for this study. 


\section{STUDY RATIONALE AND CONTEXT}

Deep vein thrombosis and pulmonary embolism are wellknown complications of lengthy orthopaedic procedures [1]. The administration of thromboprophylaxis after spine surgery is based on individual preferences [2]. It is our practice not to administer any chemical or mechanical measures, except early mobilization, to prevent thromboembolic complications [1].

\section{CLINICAL QUESTION}

What is the incidence of deep vein thrombosis (DVT) and pulmonary embolism (PE) after major spine surgery among patients receiving no DVT prophylaxis?

Fig 1 Patient sampling and selection.

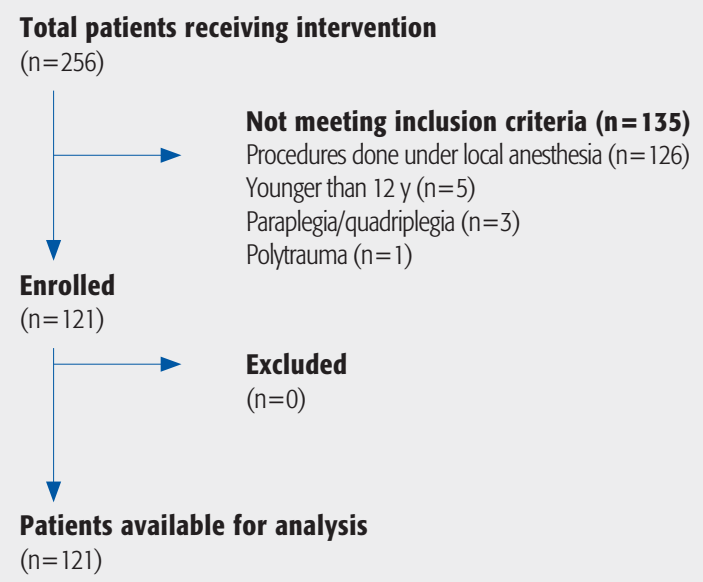

\section{METHODS}

Study design: Retrospective cohort study.

Inclusion criteria: Anterior and/or posterior spinal fusion and/or decompression of the cervical, thoracic, or lumbar region with the patient under general anesthesia (referred to as major spine procedures).

Exclusion criteria: Paraplegia, disseminated malignancy, surgery done under local anesthesia, polytrauma, younger than 12 years, and follow-up of less than 1 month.

Patient population: This study was conducted at Kovai Medical Center and Hospital, Coimbatore, India. From February 2009, 121 patients underwent major spine surgery under general anesthesia and were clinically monitored for the development of venous thrombosis or thromboembolism, from the time of admission to the last follow-up (Fig 1). No study patients were given chemoprophylaxis or stockings to prevent DVT. Every patient, their relatives, and the staff were instructed in and encouraged to begin active lower limb mobilization as soon as the patient recovered from anesthesia.

Outcomes: DVT and PE as defined by signs (tachycardia, tachypnea, and hypoxemia) and symptoms (dyspnea, swelling of the lower limbs) confirmed with Doppler scan. 


\section{RESULTS}

- One hundred and twenty-eight procedures were performed in 121 patients.

- The patient population consisted of $51 \%$ male, with a mean age of 46 years and a range of 13 to 75 years (Table 1).

- Sixty-seven percentage of the patients had comorbidities, of which hypertension $(25.6 \%)$ and diabetes mellitus $(21.5 \%)$ were the most common.

- The surgical index procedures were performed for degenerative spinal disease or intervertebral disc prolapse in $53 \%$ of patients; non-spinal cord injury (SCI)/spine fractures, 16\%; spondylolisthesis, 13\%; discovertebral osteomyelitis, $12 \%$; and deformity, $4 \%$ of cohort patients.

- The average duration of general anesthesia was 4.4 hours (range, 1-24 hours).

- Sixty-two percentage of patients had anesthetic time of 4 hours or more; and $6 \%$ had anesthetic time of more than 8 hours.

- Most of our procedures (57.8\%) were in the lumbar spine, followed by cervical spine (19.5\%), and only $4.7 \%$ were thoracic procedures.

- All patients were followed-up more than 1 month. In the outpatient department 115 (95\%) of 121 patients continued to be followed-up for more than 3 months. We had to rely on telephone-based inquiry in $6(5 \%)$ of our 121 patients for follow-up later than 1 month due to their remote home location.

- We diagnosed DVT in a single patient $(0.78 \%)$. This patient was treated successfully with anticoagulation therapy. There were no pulmonary emboli. There were no readmissions other than this patient.

- In the lumbar spine, there were 36 fusions (of which 8 were multilevel) and 33 decompression surgeries (of which 13 were multilevel).

- Anterior approaches were used in 9 cases which included 3 thoracic and 6 lumbar/thoracolumbar procedures.

- There were three combined anteroposterior surgeries.

Table 1 Patient characteristics and surgical procedures.*

\begin{tabular}{|c|c|}
\hline & No. (\%) \\
\hline \multicolumn{2}{|l|}{ Demographics ( $\mathrm{N}=121$ patients) } \\
\hline Male & $62(51.23)$ \\
\hline Mean age (range), $y$ & $46.0(13-75)$ \\
\hline Mean duration of general anesthesia (range), $\mathrm{h}$ & $4.4(1-24)$ \\
\hline \multicolumn{2}{|l|}{ Type of comorbidity } \\
\hline Hypertension & $31(25.61)$ \\
\hline Diabetes mellitus & $26(21.48)$ \\
\hline Ischemic heart disease & $6(4.95)$ \\
\hline Hypothyroidism & $5(4.13)$ \\
\hline Bronchial asthma & $5(4.13)$ \\
\hline Chronic renal failure & $3(2.47)$ \\
\hline Hypopituitarism & $1(0.82)$ \\
\hline Congenital heart block & $1(0.82)$ \\
\hline Seizure disorder & $1(0.82)$ \\
\hline Tumor (non-Hodgkin lymphoma) & $1(0.82)$ \\
\hline Rheumatoid arthritis & $1(0.82)$ \\
\hline \multicolumn{2}{|l|}{ Type of surgery ( $n=128$ procedures) } \\
\hline Lumbar & $74(57.81)$ \\
\hline Lumbar posterior laminotomy/discectomy & $33(25.78)$ \\
\hline Posterior fusion surgeries PLIF/TLIF/PLF & $36(28.12)$ \\
\hline Anterior lumbar fusions & $5(3.90)$ \\
\hline Thoracic & $6(4.68)$ \\
\hline Scoliosis posterior correction and fusion & $2(1.56)$ \\
\hline Vertebral column shortening & $3(2.34)$ \\
\hline $\begin{array}{l}\text { Scoliosis anterior release followed by second-stage posterior } \\
\text { fusion }\end{array}$ & $1(0.78)$ \\
\hline Cervical & $25(19.52)$ \\
\hline ACDF/ACF & $22(17.18)$ \\
\hline Cervical posterior procedures & $3(2.34)$ \\
\hline Thoracolumbar & $23(17.96)$ \\
\hline Posterior fracture fixation & $10(7.81)$ \\
\hline $\begin{array}{l}\text { Other posterior procedures } \\
\text { (like debridement or implant removal) }\end{array}$ & $10(7.81)$ \\
\hline Combined anterior decompression followed by posterior fusion & $3(2.34)$ \\
\hline
\end{tabular}




\section{DISCUSSION}

- There is a sustained and on-going debate in the literature regarding routine prophylaxis against venous thromboembolism in patients undergoing spine surgery [1-8]. The practices and suggestions range from chemoprophylaxis [3, 4], mechanical devices [4, 5], or no prophylaxis [6]. The incidence of postoperative venous thrombosis as such is reported to be lower in the Asian population [6]. The complications of chemoprophylaxis can be more threatening than thromboembolism itself $[3,5,6]$. Sequential compression devices [5] try to mimic the calf muscle pump [6] and hence may be superfluous if a patient actually activates the calf muscles and produces a 'pumping' effect.

- For the purpose of this study, we defined major spine surgery as one performed with the patient under general anesthesia to distinguish from minimally invasive procedures like endoscopic discectomy, which are commonly done under local anesthesia or conscious sedation in our hospital. In planning this study it was assumed that the incidence of DVT might be low after major spine surgery $[3,7]$. Therefore, no antithrombotic prophylaxes were given in order that we might determine the true incidence of venous thromboembolism after major spine surgery.

- Even though no typical primary prophylaxis was performed, the author started active range of motion exercises of both lower and upper extremities when the patient emerged from general anesthesia.

- Study strengths: This study is unique in that no prophylaxis was used in the study population, even in lengthy surgical procedures lasting more than 20 hours. It emphasizes the value of simple early mobilization, which is often forgotten, due to a sense of false security provided by chemical or mechanical thromboprophylaxis. It also relied on simple patient selection paradigm, using chemoprophylaxis only for known high-risk conditions such as patients with SCI, disseminated tumor disease, and those who were confined to bed rest for other reasons.

- Study weaknesses: We primarily relied on clinical symptoms and signs to identify our cases of DVT; therefore, some subclinical cases might have been missed [4]. But doing a Doppler study in every case is not considered cost-effective in our healthcare system considering the fact that the reported incidence of DVT is very low [5, $6,8]$. Our sample size was relatively small and consequently prevented us from identifying the incidence of the rare occurrence of PE.

\section{SUMMARY AND CONCLUSIONS}

- The duration of general anesthesia varied from 1 to 24 hours.

- No DVT prophylaxis (chemical or mechanical) was given to any selected 'low-risk' patient. Patients with SCI and disseminated neoplasia received chemical thromboembolism prophylaxis.

- Only one patient, who administered continuous epidural analgesia and was not mobilized in the early postoperative period, developed DVT. There was no clinically evident case of PE. 


\section{REFERENCES}

1. Geerts WH, Bergquist D, Pineo GF, et al (2008) Prevention of venous thromboembolism: American College of Chest Physicians, evidence-based clinical practice guidelines. (8th Edition). Chest; 133(6 Suppl):381S-453S.

2. Ploumis A, Ponnappan RK, Sarbello J, et al (2010) Thromboprophylaxis in traumatic and elective spinal surgery: analysis of questionnaire response and current practice of spinal surgeons. Spine (Phila Pa 1976); 35(3)323329.

3. Cheng JS, Arnold PM, Anderson PA, et al (2010) Anticoagulation risk in spine surgery. Spine (Phila Pa 1976); 35(9 Suppl):S117-124.

4. Committee NE-BGD (2009) Evidence-Based Clinical Guidelines for Multidisciplinary Spine Care. In: Antithrombotic Therapies in Spine Surgery. Burr Ridge, Ill: North American Spine Society.

5. Epstein NE (2006) Efficacy of pneumatic compression stocking prophylaxis in the prevention of deep venous thrombosis and pulmonary embolism following 139 lumbar laminectomies with instrumented fusions. $J$ Spinal Disord Tech; 19(1):28-31.

6. Lee HM, Suk KS, Moon SH, et al (2000) Deep vein thrombosis after major spinal surgery: incidence in an East Asian population. Spine (Phila Pa 1976); 25(14):827-1830.

7. Schuster JM, Fischer D, Dettori JR (2010) Is chemical antithrombotic prophylaxis effective in elective thoracolumbar spine surgery? Results of a systematic review. EBSJ; 1(2):41-46.

8. Smith JS, Fu KM, Polly DW Jr, et al (2010) Complication rates of three common spine procedures and rates of thromboembolism following spine surgery based on 108,419 procedures: a report from the Scoliosis Research society Morbidity and Mortality Committee. Spine (Phila Pa 1976); 35(24):2140-2149.

\section{EDITORIAL PERSPECTIVE}

Although the study is a weakly powered retrospective review of the incidence of symptomatic deep vein thrombosis (DVT) or pulmonary embolism in a consecutive series of 'major' spine surgery patients - the concept of early mobilization by the patient or the patient's family and not using other adjunctive and more costly DVT prophylaxis is an interesting idea. Tasking patients or the patients' families to reliably mobilize extremities actively or passively as soon as possible after the patient emerged from anesthesia is an example of a simple and eminently practical self-help program that could dramatically lower healthcare costs in a different litigious healthcare system. Further studies with more objective definition of type of surgery, intraoperative DVT prophylaxis, intraoperative blood loss, and resuscitation strategies, smoking status and history of coagulation disorders with more clearly delineated reporting of patient outcomes and larger numbers of patients to power the study are needed to make this a truly great article. The fact that this article can stimulate discussion, and perhaps further research into the area, gave it merit for publication in the opinion of our reviewers. 